\title{
A multicentre prospective observational study of the prevalence and glycaemic control of diabetes mellitus in adult non-cardiac elective surgical patients in hospitals in Western Cape Province, South Africa
}

\author{
T Biesman-Simons, ${ }^{1}$ MB ChB, DA (SA); W S Conradie, ${ }^{1} \mathrm{MB}$ ChB, DA (SA); M Nejthardt, ${ }^{1}$ BSc Hons (Physiology), MB BCh, \\ DA (SA), FCA (SA); F Roodt, ${ }^{1,2}$ MB ChB, FCA (SA); J Davids, ${ }^{2}$ MB ChB, DA (SA), MMed (Anaesth), FCA (SA); T Pretorius, ${ }^{3}$ MB ChB, \\ DA (SA), FCA (SA), MMed (Anaesth); G Davies, ${ }^{3} \mathrm{MB}$ ChB, FCA (SA); E Cloete, ${ }^{1,4} \mathrm{MB}$ ChB, DA (SA), FCA (SA); Z Fullerton, ${ }^{5} \mathrm{MB}$ ChB, \\ DA (SA), FCA (SA), MMed (Anaesth); J Roos, ${ }^{6}$ MB ChB, DA (SA), MMed (Anaesth), FCA (SA); M Flint, ${ }^{1}$ BSc (Medical Physiology), HSc, \\ MSc, PhD; J L Swanevelder, ${ }^{1}$ MB ChB, DA (SA), FCA (SA), MMed (Anaesth), FRCA; R A Dyer, ${ }^{1}$ MB ChB, FCA (SA), PhD, \\ B M Biccard, ${ }^{1} \mathrm{MB} \mathrm{ChB}, \mathrm{MMedSci}, \mathrm{FCA}(\mathrm{SA}), \mathrm{PhD}$
}

${ }^{1}$ Department of Anaesthesia and Perioperative Medicine, Groote Schuur Hospital and Faculty of Health Sciences, University of Cape Town, South Africa 2 Department of Anaesthesiology, George Regional Hospital, Western Cape, South Africa

${ }^{3}$ Department of Anaesthesiology, Paarl Provincial Hospital, Western Cape, South Africa

${ }^{4}$ Department of Anaesthesiology, New Somerset Hospital, Cape Town, South Africa

${ }^{5}$ Department of Anaesthesiology, Victoria Hospital, Cape Town, South Africa

${ }^{6}$ Department of Anaesthesiology, Mitchell's Plain Hospital, Cape Town, South Africa

Corresponding author: T Biesman-Simons (tbiesmansimons@gmail.com)

Background. Diabetes mellitus (DM) is a common condition. The high burden of undiagnosed DM and a lack of large population studies make accurate prevalence estimations difficult, especially in the surgical environment. Furthermore, poorly controlled DM is associated with an increased risk of perioperative complications and mortality.

Objectives. The primary objective was to establish the prevalence of DM in elective adult non-cardiac, non-obstetric surgical patients in hospitals in Western Cape Province, South Africa. The secondary objectives were to assess the glycaemic control and compliance with treatment of known diabetics.

Methods. A 5-day multicentre, prospective observational study was performed at six government-funded hospitals in the Western Cape. Screening for DM was done using finger-prick capillary blood glucose (CBG) testing. Patients found to have a CBG $\geq 6.5 \mathrm{mmol} / \mathrm{L}$ had their glycated haemoglobin (HbAlc) level measured. DM was diagnosed based on the Society for Endocrinology, Metabolism and Diabetes of South Africa (SEMDSA) diagnostic criteria. Patients known to have DM had their HbAlc measured and completed a Morisky Medication Adherence Scale (MMAS-4) questionnaire to assess glycaemic control and compliance with treatment.

Results. Of the 379 participants, 61 were known diabetics (16.2\%; 95\% confidence interval (CI) $12.4-19.8$ ). After exclusion of 8 patients with incomplete results, a new diagnosis of DM was made in 5/310 patients (1.6\%; $95 \%$ CI $0.2-3.0)$. The overall prevalence of DM was 17.8\% (66/371; 95\% CI 13.9 - 21.7). HbA1c results were available for 57 (93.4\%) of the 61 known diabetics. Of these, 27 (47.4\%; 95\% CI 34.4 - 60.3) had an HbAlc level $\geq 8.5 \%$ and 14 (24.6\%; 95\% CI $13.4-35.8)$ had a level $\leq 7 \%$. Based on positive responses to two or more questions on the MMAS-4 questionnaire, $12 / 60$ participants (20.0\%) were deemed non-compliant.

Conclusions. There is a low rate of undiagnosed DM in our elective surgical population, but in a high proportion of patients with DM the condition is poorly controlled. Poorly controlled DM is known to increase postoperative complications and is likely to increase the burden of perioperative care. Resources should be focused on improvement of long-term glycaemic control in patients presenting for elective surgery.

S Afr Med J 2019;109(10):801-806. https://doi.org/10.7196/SAMJ.2019.v109i10.13898

Diabetes mellitus (DM) is a common condition, affecting an estimated 15.5 million people in Africa. Importantly, the prevalence of DM across the continent is expected to double by $2045 .^{[1]}$ Since 2015 , this condition has been ranked as the second most common cause of natural death in South Africa (SA), and its impact on healthcare provision is substantial. ${ }^{[2]}$ Accurate assessment of prevalence is difficult owing to the high burden of undiagnosed DM (estimated at $69 \%$ in Africa) and the lack of large population studies. ${ }^{[1]}$ In SA, the prevalence of DM is estimated to be between $5.4 \%$ and $9.2 \%{ }^{[1,3]}$ There are limited data reporting the prevalence of DM in Western Cape Province, SA, and information with regard to elective surgical patients is minimal. Many studies have shown that DM, especially if poorly controlled, is associated with an increased risk of perioperative complications and mortality. ${ }^{[4-9]}$ In SA, insulin-dependent surgical patients are twice as likely as non-diabetics to die in hospital. ${ }^{[10]}$

\section{Objectives}

The primary objective of this study was to establish the prevalence of DM in patients presenting for elective surgery over a 1-week period in six Western Cape hospitals. This included patients with a previous diagnosis of DM, and those with a new diagnosis based on screening capillary blood glucose $(\mathrm{CBG})$ testing and a confirmatory elevated glycated haemoglobin $(\mathrm{HbAlc})$ level. The secondary objectives were to assess: $(i)$ the glycaemic control of known diabetics presenting for 
surgery, using the HbAlc level; and (ii) compliance with treatment, with the Morisky Medication Adherence Scale (MMAS-4). ${ }^{[11]}$

\section{Methods}

Study approval was obtained from the University of Cape Town Faculty of Health Sciences Human Research Ethics Committee (ref. no. UCT HREC: 386/2017) and the Western Cape Department of Health, and institutional approval was provided by all participating centres (ref. no. NHRD:WC_201709_018). The study was registered on Clinicaltrials.gov (ref. no. NCT03318055). Written informed consent was provided by all participants prior to enrolment. The study is presented according to the Strengthening the Reporting of Observational Studies in Epidemiology (STROBE) guidelines. ${ }^{[12]}$

This was a multicentre, prospective observational study performed at six government-funded hospitals in the Western Cape: Groote Schuur, New Somerset, Paarl, Victoria, Mitchell's Plain and George hospitals. A prestudy power calculation based on an expected prevalence of DM of $10 \%^{[10]}$ and an estimated sample size of 500 (expected number of elective surgical procedures in the participating hospitals) allowed for an estimate of the prevalence with a $95 \%$ confidence interval (CI) of $\pm 2.65 \%$, i.e. 7.35 - $12.65 \%$. Convenience sampling was done during the daytime hours $(07 \mathrm{~h} 00$ - 19h00) of a calendar week (Monday 16 October - Friday 20 October 2017).

Exclusion criteria were patient refusal or inability to consent, emergency and cardiac surgery, and pregnant and paediatric $(<18$ years of age) patients. All participants were seen preoperatively by the anaesthesia medical staff, and after written consent had been obtained, demographic and baseline data were collected and recorded on the case report form (CRF) (available as a supplementary file at http://www.samj.org.za/public/sup/simons_13898.pdf).

The diagnosis of DM in our study was based on recommendations of the Society for Endocrinology, Metabolism and Diabetes of South Africa (SEMDSA), ${ }^{[13]}$ which state that the diagnosis can be made as follows: in an asymptomatic patient when any one of the following tests, repeated on separate days within a 2-week period, is confirmed: (i) fasting plasma glucose $\geq 7.0 \mathrm{mmol} / \mathrm{L}$; (ii) 2 hours post glucose load $\geq 11.1 \mathrm{mmol} / \mathrm{L}$; or (iii) $\mathrm{HbAlc} \geq 6.5 \%$. In the event that both a glucosebased test and the HbAlc test are done, if both are 'diagnostic' for diabetes, the diagnosis is confirmed.

$\mathrm{HbAlc}$ was measured in known diabetics who had not had a test result within the preceding 90 days. Screening for DM was done using finger-prick CBG testing in all consenting participants undergoing elective surgery. The mandatory starvation period of at least 6 hours for elective surgical patients was used as the fasting period for the fasting glucose measurement. Based on the recommended correction of CBG to reflect true plasma glucose (plasma glucose $(\mathrm{mmol} / \mathrm{L})=$ $0.102+1.066 \times \mathrm{CBG}),{ }^{[13]}$ a capillary glucose level of $\geq 6.5 \mathrm{mmol} / \mathrm{L}$ was taken as a cut-off to reflect a plasma level $\geq 7.0 \mathrm{mmol} / \mathrm{L}$. Patients found to have a CBG of $\geq 6.5 \mathrm{mmol} / \mathrm{L}$ had an $\mathrm{HbAlc}$ test to confirm the diagnosis of diabetes.

$\mathrm{HbAlc}$ results were linked via a unique CRF-generated laboratory number. Data were recorded on paper CRFs, and captured electronically onto the Research Electronic Data Capture (REDCap) application. Access to REDCap was protected by username and password. Patient confidentiality and anonymity were protected through unique numerical code generation during electronic data capturing. Patients identified as having raised fasting CBG were given referral letters to their primary healthcare facilities for further investigations and management.

SEMDSA advocates aiming for an HbAlc level of $\leq 7 \%$ in most patients (well-controlled DM). An HbA1c level of $7.1-8.5 \%$ is considered to indicate moderate control and may be acceptable in the following patient categories: elderly, frail, limited life expectancy, multiple comorbidities, severe vascular disease, advanced chronic kidney disease, recurrent severe hypoglycaemia, or unawareness of hypoglycaemia. ${ }^{[13]}$ An HbAlc level of $\geq 8.5 \%$ is considered to indicate poor control. Compliance with treatment was assessed using the MMAS-4 score. ${ }^{[11]}$ Non-compliance was taken as a positive response to two or more of the four questions in the MMAS-4.

\section{Statistical analysis}

Continuous variables with normal distribution were described as means and standard deviations (SDs). Between-group comparisons were performed using Fisher's exact test, except for age ( $t$-test) and in contingency tables that were more than $2 \times 2$ comparisons (American Society of Anesthesiologists (ASA) classification and functional status), where Pearson's $\chi^{2}$ test was used. The Statistical Package for the Social Sciences (SPSS) version 24 (SPSS Inc., USA) was used for data analysis.

\section{Results}

The study flow diagram is shown in Fig. 1, and the breakdown of the contribution to final participant numbers from each hospital in Table 1. Participants known to have DM comprised 16.1\% (61/379; 95\% CI 12.4 - 19.8) of the study population. Of the 318 participants who had not previously been diagnosed with DM, 310 (97.5\%) had CBG and, where appropriate, HbAlc results available. A new diagnosis of DM was made in an additional 1.6\% (5/310; 95\% CI 0.2 3.0) of participants not previously known to have DM. The overall prevalence of DM was therefore $17.8 \%$ (66/371; 95\% CI 13.9 - 21.7). Of the 66 diabetic patients, $7.5 \%(5 / 66 ; 95 \%$ CI 1.2 - 13.9) were diagnosed during the study.

The demographic details of the study population are shown in Table 2. Univariate analysis showed an association between DM and increasing age, increasing ASA physical status classification, decreasing functional status and comorbidities. Based on positive responses to two or more questions on the MMAS-4 questionnaire, $12 / 60$ participants (20.0\%) were non-compliant with therapy for DM.

\section{Discussion}

\section{Principal findings}

In our study population, one in six participants presenting for surgery was diabetic. Of the participants with DM, 92.5\% were known diabetics prior to surgery, but almost half had poor glycaemic control as reflected by an HbAlc level $\geq 8.5 \%$ (Table 3 ).

The strength of this investigation was that it was a multicentre, prospective study that involved several regions in the Western Cape, SA. The research provided the most comprehensive data to date of the prevalence of diabetes in this elective adult surgical patient population, and therefore has implications for determining appropriate management plans for this population. In comparison with some previous prevalence studies, the study did not rely solely on self-reporting of DM or single CBG results, and we therefore believe that it is a more accurate representation of the true prevalence of diabetes and the degree of glycaemic control in these elective surgical patients.

\section{Relation to other studies}

The prevalence of DM in our study was higher than reported in non-surgical SA population studies. The International Diabetes Federation country-level DM estimates (5.4\% for SA) were based on the weighted average of the scores of all data sources in the adult population. ${ }^{[1]}$ The South African National Health and Nutrition 


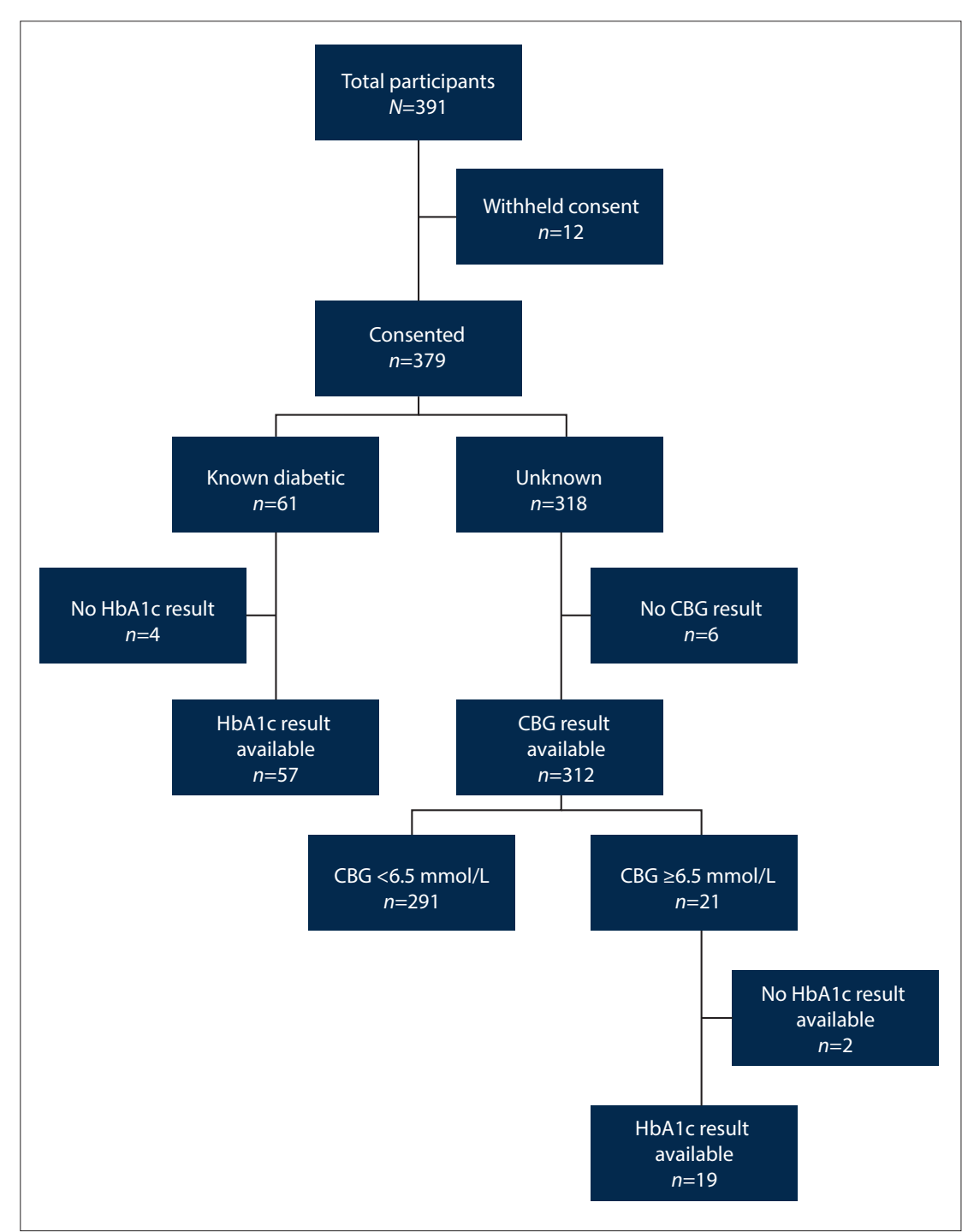

Fig. 1. Study flow diagram. (HbA1c = glycated haemoglobin; $C B G=$ capillary blood glucose. $)$

\begin{tabular}{ll} 
Table 1. Participating hospitals \\
\hline Hospital & $\begin{array}{l}\text { Participants } \\
(\boldsymbol{N}=\mathbf{3 7 9}), \boldsymbol{n}\end{array}$ \\
\hline Groote Schuur & 176 \\
George & 58 \\
Paarl & 42 \\
New Somerset & 40 \\
Victoria & 35 \\
Mitchell's Plain & 28
\end{tabular}

Examination Survey (SANHANES) utilised a multistage disproportionate, stratified cluster sampling approach to the general population based on census data, and demonstrated a $9.2 \%$ prevalence of $\mathrm{DM}$ (self-reported, and $\mathrm{HbAlc} \geq 6.5 \%$ ) in the adult population. ${ }^{[3]}$ The prevalence of DM increases with age, and the higher mean age of our study population than that of the general SA population probably contributes to the higher prevalence of DM reported. ${ }^{[14]}$
Studies of surgical patients report a higher prevalence of DM than in the population overall. The South African Surgical Outcomes Study reported an SA national prevalence of self-reported DM of $10.1 \%{ }^{[10]}$ This figure is considerably lower than ours. When investigating elective surgical patients in the Western Cape, Van der Spuy et al. ${ }^{[15]}$ showed a prevalence of self-reported DM of $16 \%$, which is in keeping with our findings. However, neither of these studies evaluated the prevalence of undiagnosed DM at the time of surgery, or the degree of glycaemic control.

Using CBG and HbAlclevels as screening and diagnostic tools, our study identified a low prevalence of undiagnosed DM of 1.6\% $(5 / 310)$ in this surgical population. This is a considerably lower figure than that reported in a general population study by Bailey et al., ${ }^{[16]}$ which estimated a prevalence of undiagnosed DM (based on random CBG measurement) in the Western Cape of $12.7 \%$. In 2012, Erasmus et al. ${ }^{[17]}$ found a prevalence of undiagnosed DM (based on oral glucose tolerance tests and $\mathrm{HbAlc}$ ) of $18.2 \%$ in adults in a community in Bellville, Western Cape. The reasons for the lower prevalence in our study probably include: (i) the stringent diagnostic criteria we applied; and (ii) the fact that all participants in our study had been assessed preoperatively by a number of healthcare practitioners.

\section{Clinical implications}

Our data suggest that DM screening for patients accessing elective surgery (often via primary healthcare) is well established in the studied healthcare services. Based on our findings, we suggest that screening for DM is not a priority in the preoperative period. This proposal is in keeping with conclusions from a systematic review that routine blood glucose or HbAlc levels are not needed in otherwise well non-diabetic patients presenting for general (non-orthopaedic or non-vascular) surgery. ${ }^{[18]}$ However, cognisance must be taken of the fact that a small number of patients do present with undiagnosed DM. The prevalence of DM and access to primary healthcare and hospitals are extremely variable in SA, and care must be taken in generalising our findings over too broad a population base.

Numerous studies have shown that poor preoperative glycaemic control is associated with increased complication rates in the perioperative period. These include increased length of stay, ${ }^{[4]}$ an increased risk of failure of total ankle replacements, ${ }^{[5]}$ increased infection rates in hip and knee arthroplasty, ${ }^{[6]}$ and an increased risk of poor postoperative glycaemic control and postoperative complications. ${ }^{[7]}$ In 2015, Kallio et al. ${ }^{[19]}$ demonstrated that referral of poorly controlled diabetic patients (HbA1c $>10 \%)$ to primary healthcare services for optimisation of glycaemic control before proceeding with total joint arthroplasty resulted in lower complication rates and shorter hospital stays.

The UK National Health Service Consensus Guidelines of $2011,{ }^{[20]}$ endorsed by the Association of Anaesthetists of Great Britain and Ireland Working Party, ${ }^{[21]}$ recommend postponing elective surgery in patients with an HbA1c level $\geq 8.5 \%$, to allow for improved glycaemic control. In our study, $47 \%$ of diabetic participants had an HbAlc exceeding this level. If these guidelines were to be adopted in the Western Cape, it is likely that a significant proportion of diabetic patients would have their surgery postponed. 
Table 2. Characteristics of the study population

\begin{tabular}{|c|c|c|c|c|}
\hline & Patients $(N=371)$ & Diabetic $(N=66,17.8 \%)$ & Non-diabetic $(N=305,82.2 \%)$ & $p$-value \\
\hline Age (years), mean (SD) & $50.6(16.5)$ & $60.0(11.9)$ & $48.6(16.7)$ & $<0.001$ \\
\hline Male gender, $n / N(\%)$ & $133 / 371(36.4)$ & $22 / 66(33.3)$ & $113 / 305$ & 0.672 \\
\hline Major surgery, $n / N(\%)$ & 40/371 (10.8) & $5 / 66(7.6)$ & $35 / 305(11.5)$ & 0.511 \\
\hline ASA classification, $n / N(\%)$ & & & & $<0.001$ \\
\hline ASA 1 & 90/368 (24.5) & $0 / 65(0)$ & $90 / 303(29.7)$ & \\
\hline ASA 2 & $193 / 368(52.4)$ & $32 / 65(49.2)$ & $161 / 303(53.1)$ & \\
\hline ASA 3 & $80 / 368(21.7)$ & $29 / 65(44.6)$ & $51 / 303(16.8)$ & \\
\hline ASA 4 & $5 / 368(1.4)$ & $4 / 65(6.2)$ & $1 / 303(0.3)$ & \\
\hline \multicolumn{5}{|l|}{ Co-morbid conditions, $n / N(\%)$} \\
\hline Smoker & $128 / 367(34.9)$ & $16 / 66(24.2)$ & $112 / 301(37.2)$ & 0.047 \\
\hline History of coronary artery disease & $17 / 371(4.6)$ & $7 / 66(10.6)$ & $10 / 305(3.3)$ & 0.018 \\
\hline History of congestive heart failure & $5 / 371(1.3)$ & $3 / 66(4.5)$ & $2 / 305(0.7)$ & 0.041 \\
\hline Stroke/TIA & $15 / 371(4.0)$ & $8 / 66(12.1)$ & $7 / 305(2.3)$ & 0.002 \\
\hline Chronic renal disease & $18 / 371(4.9)$ & $7 / 66(10.6)$ & $11 / 305(3.6)$ & 0.026 \\
\hline Peripheral arterial disease & $3 / 371(0.8)$ & $2 / 66(3.0)$ & $1 / 305(0.3)$ & 0.083 \\
\hline Hypertension & $165 / 371(44.5)$ & $55 / 66(83.3)$ & $110 / 305(36.1)$ & $<0.001$ \\
\hline Advanced retinopathy & $13 / 371(3.5)$ & $13 / 66(19.7)$ & $0 / 305(0)$ & $<0.001$ \\
\hline Functional status, $n / N(\%)$ & & & & $<0.001$ \\
\hline Totally independent & $324 / 370(87.6)$ & $46 / 66(69.7)$ & 278/304 (91.4) & \\
\hline Partially dependent & $39 / 370(10.5)$ & $19 / 66(28.8)$ & $20 / 304(6.6)$ & \\
\hline Totally dependent & $7 / 370(1.9)$ & $1 / 66(1.5)$ & $6 / 304(2.0)$ & \\
\hline
\end{tabular}

Table 3. Glycaemic control of known diabetics based on HbAlc results

\begin{tabular}{llll}
\hline & HbA1c $\leq 7 \%$ & HbA1c 7.1 - 8.4\% & HbA1c $\geq 8.5 \%$ \\
\hline Known diabetics, $n(\% ; 95 \%$ CI $)$ & $14(24.6 ; 13.4-35.8)$ & $16(28.1 ; 16.4-39.7)$ & $27(47.4 ; 34.4-60.3)$ \\
HbAlc = glycated haemoglobin; $\mathrm{CI}=$ confidence interval. & & &
\end{tabular}

In our resource-limited environment, patients may experience long delays in accessing elective surgery. Last-minute postponement of surgery to allow for improved glycaemic control may not be practical. If poor control is identified timeously, the long waiting period presents an opportunity for optimisation of treatment without increasing the delay before surgery. Limited access to good-quality diabetes care is a major concern: an analysis of data from the SANHANES study estimated that only $19.4 \%$ of patients with DM in SA are both identified and well controlled, ${ }^{[22]}$ which is in keeping with our findings, where only $24.6 \%$ of patients known to have DM had good control ( $\mathrm{HbAlc} \leq 7 \%)$.

As a secondary outcome, compliance was assessed using the MMAS-4. Only one of every five participants was noted to be noncompliant, which is similar to the prevalence of self-reported noncompliance with DM medication of $30 \%$ in Limpopo Province. ${ }^{[23]}$ In our study, no conclusions could be drawn with regard to the correlation between compliance and glycaemic control. Verbal questionnaires to establish compliance have limited reliability. We used the MMAS- 4 because this tool has been described as nearest to the gold standard. ${ }^{[24]}$ Despite the reported compliance rate of $80 \%$ in our study population, $47.4 \%$ of the diabetic participants were found to be poorly controlled, with an $\mathrm{HbAlc}$ level of $\geq 8.5 \%$. Interpreting the reasons for the disparity between reported medication compliance and glycaemic control is complex. Factors contributing to glycaemic control may include the prescribing and availability of correct medication, as well as lifestyle factors such as exercise and diet.

\section{Study limitations}

This study has some limitations. A smaller number of patients was recruited than we expected ( 379 v. 500 ) owing to the withdrawal of one of the hospitals initially planned to be an active site. This minimally increased the $95 \%$ CI associated with estimation of the prevalence of diabetes in this population from 5.3 to 8 .

Nil per os guidelines followed in the participating hospitals were a minimum of 6 hours for solid food and 2 hours for clear fluids. In practice, most patients had longer nil per os times than this minimum requirement. The SEMDSA guidelines state that 'fasting is defined as no caloric intake for at least eight hours. ${ }^{[13]}$ It is possible that participants may have had oral or intravenous glucose containing fluids within 8 hours preoperatively, which may have affected the CBG result. Fasting status would not affect HbA1c results. Since $\mathrm{HbAlc}$ was used as a defining result for DM, we are confident that we did not overestimate the prevalence of DM in our study population.

The SEMDSA guidelines advise that bedside tests (glucose and HbA1c) should not be used to diagnose DM unless laboratory-based tests are unavailable. ${ }^{[13]}$ For reasons of clinical convenience, and to make our study practicable, we used finger-prick CBG levels. We conducted laboratory $\mathrm{HbAlc}$ testing as a defining result to diagnose DM. The SEMDSA guidelines also state that if only one of these tests is abnormal, a second abnormal result of the same testing method is required to confirm the diagnosis of diabetes on a different day, preferably within two weeks. ${ }^{[13]}$ Fourteen of our participants had a raised CBG level, with an $\mathrm{HbA1c}$ level $<6.5 \%$, and were not followed 
up. Our results may therefore underestimate the prevalence of undiagnosed diabetes in this surgical population.

\section{Suggested further research}

This study provides an objective assessment of the prevalence and glycaemic control of DM in the perioperative patient population in the Western Cape, SA. The poor control of DM in the perioperative period suggests that further research is needed to evaluate perioperative complication rates in these patients. Interventions to improve longterm glycaemic control should be identified in elective surgical patients, and the effects of their introduction should be studied.

\section{Conclusions}

$\mathrm{DM}$ is a common disease that is associated with increased perioperative complications. It was well diagnosed but poorly managed in our study population. We recommend that early identification of poorly managed DM (by HbA1c measurement) should be prioritised in elective surgical patients. Doing this could result in timeous referral to the appropriate services for improvement of glycaemic control before surgery, and in turn allow time for improvement of preoperative management of DM, without causing a significant increase in surgical waiting times. Overall, the goal would be lower perioperative complication rates.

Declaration. This study reported on was part of the requirements of TB-S's MMed (Anaesth) degree.

Acknowledgements. We acknowledge and thank all the anaesthetic medical officers, registrars and consultants involved in the study design, planning and organisation, as well as all who were involved in data collection and capture at the various institutions included in the study: George Hospital, Groote Schuur Hospital, Mitchell's Plain Hospital, New Somerset Hospital, Paarl Hospital and Victoria Hospital. We also thank the Department of Anaesthesia and Perioperative Medicine at Groote Schuur Hospital for accommodating the roster and other logistic changes necessary to make this study possible.

Author contributions. This submission has 14 authors from a multicentre, prospective observational study of six hospitals in the Western Cape, SA. We have itemised their contributions according to the International Committee of Medical Journal Editors criteria. TB-S: overall conception and design of the study, acquisition of data at Groote Schuur Hospital, interpretation, drafting and critical revising of the work, final approval of the version to be published, agree to be accountable for all aspects, accuracy and integrity of the work; WSC: overall conception and design of the study, acquisition of data at Groote Schuur Hospital, interpretation, critical revising of the work, final approval of the version to be published, agree to be accountable for all aspects, accuracy and integrity of the work; MN: overall conception and design of the study, analysis and interpretation, drafting and critical revising of the work, final approval of the version to be published, agree to be accountable for all aspects, accuracy and integrity of the work; FR: overall conception and design of the study, analysis and interpretation, critical revising of the work, agree to be accountable for all aspects, accuracy and integrity of the work; JD: acquisition of data at George Hospital, critical revising of the work, final approval of the version to be published, agree to be accountable for all aspects, accuracy and integrity of the work; TP: acquisition of data at Paarl Hospital, critical revising of the work, final approval of the version to be published, agree to be accountable for all aspects, accuracy and integrity of the work; GD: acquisition of data at Paarl Hospital, critical revising of the work, final approval of the version to be published, agree to be accountable for all aspects, accuracy and integrity of the work; EC: acquisition of data at New Somerset Hospital, critical revising of the work, final approval of the version to be published, agree to be accountable for all aspects, accuracy and integrity of the work; ZF: acquisition of data at Victoria Hospital, critical revising of the work, final approval of the version to be published, agree to be accountable for all aspects, accuracy and integrity of the work; JR: acquisition of data at Mitchell's Plain Hospital, critical revising of the work, final approval of the version to be published, agree to be accountable for all aspects, accuracy and integrity of the work; MF: acquisition of data at Groote Schuur Hospital, critical revising of the work, final approval of the version to be published, agree to be accountable for all aspects, accuracy and integrity of the work; JLS: overall conception and design of the study, critical revising of the work, final approval of the version to be published, agree to be accountable for all aspects, accuracy and integrity of the work; RAD: overall conception and design of the study, critical revising of the work, final approval of the version to be published, agree to be accountable for all aspects, accuracy and integrity of the work; BMB: overall conception and design of the study, analysis of results, drafting and critical revising of the work, final approval of the version to be published, agree to be accountable for all aspects, accuracy and integrity of the work.

Funding. Funding for this study was received from the South African Society of Anaesthetist's Jan Pretorius Research Fund. Resources of the Department of Anaesthesia and Perioperative Medicine at Groote Schuur Hospital were utilised.

Conflicts of interest. None.

1. International Diabetes Federation. IDF Diabetes Atlas 8th Edition 2017. http://www.diabetesatlas.or (accessed 16 June 2018).

2. Statistics South Africa. Media Release: Mortality and causes of death 2015. 2017. www.statssa.gov.za. http://www.statssa.gov.za/?p=9604 (accessed 27 September 2018).

3. Shisana O, Labadarios D, Rehle T, et al. South African National Health and Nutrition Examination Survey, 2012: SANHANES-1: The Health and Nutritional Status of the Nation. Cape Town: Human Sciences Research Council, 2014

4. Underwood P, Askari R, Hurwitz S, et al. Preoperative AlC and clinical outcomes in patients with diabetes undergoing major noncardiac surgical procedures. Diabetes Care 2014;37(3):611-616. https:// doi.org/10.2337/dc13-1929

5. Choi WJ, Lee JS, Lee M, et al. The impact of diabetes on the short- to mid-term outcome of total ankl replacement. Bone Joint J 2014;96-B(12):1674-1680. https://doi.org/10.1302/0301-620x.96b12.34364

6. Shohat N, Muhsen K, Gilat R, et al. Inadequate glycemic control is associated with increased surgical site infection in total joint arthroplasty: A systematic review and meta-analysis. J Arthroplasty 2018;33(7):2312-2321. https://doi.org/10.1016/j.arth.2018.02.020

7. Gustafsson UO, Thorell A, Soop M, et al. Haemoglobin Alc as a predictor of postoperative hyperglycaemia and complications after major colorectal surgery. Br J Surg 2009;96(11):1358-1364. hyperglycaemia and complications

8. Yeh CC, Liao CC, Chang YC, et al. Adverse outcomes after noncardiac surgery in patients with diabetes: A nationwide population-based retrospective cohort study. Diabetes Care 2013;36(10):3216-3221. A nationwide population-based
https://doi.org $/ 10.2337 /$ dc13-0770

9. Pearse RM, Rhodes A, Moreno R, et al. EuSOS: European Surgical Outcomes Study. Eur J Anaesthesiol Pearse RM, Rhodes A, Moreno R, et al. EuSOS: European Surgical Outco
2011;28(6):454-456. https://doi.org/10.1097/EJA.0b013e328344907b

10. Biccard BM, Madiba TE. The South African Surgical Outcomes Study: A 7-day prospective observational cohort study. S Afr Med J 2015;105(6):465-475. https://doi.org/10.7196/SAMJ.9435

11. Morisky DE, Green LW, Levine DM. Concurrent and predictive validity of a self-reported measure of medication adherence. Med Care 1986;24(1):67-74. https://doi.org/10.1097/00005650-198601000-00007

12. Von Elm E, Altman DG, Egger M, et al. The Strengthening the Reporting of Observational Studies in Epidemiology (STROBE) Statement: Guidelines for reporting observational studies. Int J Surg 2014;12(12):1495-1499. https://doi.org/10.1016/j.ijsu.2014.07.013

13. Society for Endocrinology, Metabolism and Diabetes of South Africa Type 2 Diabetes Guidelines Expert Committee. The 2017 SEMDSA Guidelines for the Management of Type 2 Diabetes. JEMDSA 2017:22(1, Suppl 1):S1-S196. http.//wwwjemdsaco za/index php/IEMDSA/article/view/647 (accessed 27 Aun 201722(1,

Suppl 1):S1-S196. http://www.jemdsa.co.za/index.php/JEMDSA/article/view/647 (accessed 27 August 2019). Statistics South Africa. Mid-year population estimates 2017. Statistical release P0302. Pretoria: Stats S 2017. https://www.statssa.gov.za/publications/P0302/P03022017.pdf (accessed 27 September 2018).

15. Van der Spuy K, Crowther M, Nejthardt M, et al. A multicentre, cross-sectional study investigating the prevalence of hypertensive disease in patients presenting for elective surgery in the Western Cape Province, South Africa. S Afr Med J 2018;108(7):590-595. https://doi.org/10.7196/SAMJ.2018. v108i7.13022

16. Bailey SL, Ayles H, Beyers N, et al. Diabetes mellitus in Zambia and the Western Cape province of South Africa: Prevalence, risk factors, diagnosis and management. Diabetes Res Clin Pract 2016;118:1-11. https://doi.org/10.1016/j.diabres.2016.05.001

17. Erasmus RT, Soita DJ, Hassan MS, et al. High prevalence of diabetes mellitus and metabolic syndrome in a South African coloured population: Baseline data of a study in Bellville, Cape Town. S Afr Med J 2012;102(11):841-844. https://doi.org/10.7196/SAMJ.5670

18. Bock M, Johansson T, Fritsch G, et al. The impact of preoperative testing for blood glucose concentration and haemoglobin Arc on mortality, changes in management and complications in noncardiac eja.0000000000000117 
19. Kallio PJ, Nolan J, Olsen AC, et al. Anesthesia preoperative clinic referral for elevated HbAlc reduces complication rate in diabetic patients undergoing total joint arthroplasty. Anesth Pain Med 2015;5(3):e24376. https://doi.org/10.5812/aapm.5(3)2015.24376

20. Dhatariya K, Levy N, Kilvert A, et al. NHS diabetes guideline for the perioperative managemen of the adult patient with diabetes. Diabet Med 2012;29(4):420-433. https://doi.org/10.1111/j.14645491.2012.03582.x

21. Barker P, Creasey PE, Dhatariya K, et al. Peri-operative management of the surgical patient with diabetes 2015: Association of Anaesthetists of Great Britain and Ireland. Anaesthesia 2015;70(12):1427-1440. https://doi.org/10.1111/anae.13233

22. Stokes A, Berry KM, McHiza Z, et al. Prevalence and unmet need for diabetes care across the care continuum in a national sample of South African adults: Evidence from the SANHANES-1, 2011 - 2012 PLoS One 2017;12(10):e0184264. https://doi.org/10.1371/journal.pone. 0184264
23. Adegbola SA, Marincowitz GJ, Govender I, et al. Assessment of self-reported adherence among patients with type 2 diabetes in Matlala District Hospital, Limpopo Province. Afr J Prim Health Care Fam Med 2016:8(1):el-5. https://doi.org/10.4102/phcfm.v8i1.900

24. Culig J, Leppee M. From Morisky to Hill-bone: Self-reports scales for measuring adherence to medication. Coll Antropol 2014;38(1):55-62.

Accepted 22 March 2019 